\title{
SELECTED PROPERTIES OF LATTICE DYNAMICS OF HgSe AND $\beta$-HgS*
}

\author{
W. Szuszkiewicz, K. Dybko, E. Dynowska, J. Górecka, B. Witkowska \\ Institute of Physics, Polish Academy of Sciences \\ Al. Lotników 32/46, 02-668 Warszawa, Poland
}

B. HENNION

Laboratoire Léon Brillouin ${ }^{\dagger}$, CE Saclay, 91191 Gif-sur-Yvette cédex, France

\author{
M. Jouanne and C. Julien
}

Laboratoire des Milieux Désordonnés et Hétérogènes, Université Pierre et Marie Curie Case 86, 4, place Jussieu, 75252 Paris cédex 05, France

Optical phonon dispersion for $\beta$-HgS was measured by the neutron scattering for the first time. The results confirmed theoretically predicted anomalous behavior of phonon modes in this material, resulting probably from high ionicity of mercury sulphide and large difference of $\mathrm{Hg}$ and $\mathrm{S}$ atomic mass. Influence of the isotopic effects on the TO-phonon mode for $\mathrm{HgSe}$ and $\mathrm{HgS}$ is analyzed. The possible observation of such effects in IR reflectivity spectra taken for HgSe at low temperature is discussed.

PACS numbers: 61.12.-q, 63.20.-e, 78.30.-j

The goal of this letter was to signalize the new interesting features, found in the lattice dynamics of mercury chalcogenides, studied by three independent methods: IR reflectivity, Raman scattering and neutron scattering measurements. All investigated crystals were grown using a modified Bridgman method. In the case of HgSe both, pure compound and Co or Fe doped materials were used for the optical measurements. The cubic mercury sulphide $(\beta-\mathrm{HgS})$ is a metastable modification of this semiconductor which cannot be obtained in a bulk form by the equilibrium growth methods. Monocrystals of this compound in the zinc blende structure were obtained by heavy doped $\mathrm{HgS}$ with transition metal impurities [1]. Such samples were used for the IR reflectivity and Raman scattering measurements. Finally, the neutron scattering measurements were performed on $\mathrm{HgS}$ containing about $2 \%$

*This work is supported in part by PB 177/P03/96/11 grant from the Committee for Scientific Research (Poland) and by the European Community Program Human Capital and Mobility Access to Large Scale Facilities PECO Extension (contract No. ERB CIPD CT 940080).

†Laboratoire commun CEA-CNRS. 
of $\mathrm{Fe}$ and on pure $\mathrm{HgSe}$. The crystal quality was checked by the X-ray diffraction measurements. In the case of doped materials the chemical composition was determined by the energy dispersive X-ray fluorescence (EDXRF) analysis. The composition was also estimated from the lattice parameter value measurements.

Inelastic neutron scattering experiments were performed at Orphee reactor in Laboratoire Léon Brillouin (LLB) in Saclay on a triple-axis spectrometer installed on a thermal source. The monochromator as well as the analyzer were pyrolytic graphite oriented in (002) reflection. All scans were performed with constant final wave vector $q=2.662 \AA^{-1}$. Because of the expected non-monotonous dependence of optical phonon frequency on the phonon wave vector, the HgSe and $\beta$-HgS optical branch dispersion was of the particular interest (the optical branch for HgTe, which does not change a lot, was shown in [2]). The optical phonon dispersion curves for HgSe determined along the high symmetry directions (001) and (011) could be found in [3]. First results concerning the optical branches of $\beta$-HgS obtained on the basis of the neutron scattering measurements are shown in Fig. 1. The predicted high dispersion of TO phonon mode and an increase in LO phonon frequency with the increasing wave vector value [4] were confirmed by the neutron scattering measurements. As we believe this phenomenon is related to both high ionicity of material and large difference between $\mathrm{Hg}$ and $\mathrm{S}$ mass (constituent elements mass ratio higher than 6 ) and results from the particular set of model [5] parameter values. New neutron scattering data enable us also to explain a very rich structure observed previously in the Raman scattering spectra [6]. In particular, an important role of the two-phonon modes with the participation of TA phonons was found (this problem will be discussed in detail elsewhere [7]).

All investigated samples were $n$-type materials with a high concentration of free electrons which provide the TO-phonon frequency well below the plasma edge. Nevertheless, it was possible to investigate the small phonon-related structures in the metallic reflectivity spectra by means of the Fourier spectrometer. It was found that using the instrument resolution better than $2 \mathrm{~cm}^{-1}$ this structure is asymmetric for HgSe but seems to be still symmetric for $\beta$-HgS. In order to explain this behavior the isotope effects (related to a few isotopes present in natural element) were taken into consideration. The possible influence of the isotope effects on the frequency and the shape of TO-phonon related structure was roughly estimated within the rigid ion model (under assumption that the force constants do not differ for various isotopes and the only difference is due to the atomic mass). The results show that in the simplest possible approximation one could expect some frequency separation between the modes corresponding to the different isotopes.

Comparing the reflectivity spectra taken for $\mathrm{HgSe}$ and $\beta$-HgS (both with similar concentration of $\mathrm{Fe}$ impurities and free-carrier concentration) it was demonstrated that isotope effects could really influence the observed shape of TO-phonon related structure. Figure $2 a$ shows an idea which one could expect when analyzing the TO phonon mode structure for HgSe. Selenium has five principal stable isotopes. Vibrations of Se atoms give the principal contribution to the optical phonon branch. In the first (rather rough) approximation slightly different vibration frequency corresponds to each isotope. In our neutron or optical experiment such contributions could not be resolved. Nevertheless, as one can see, the shape of 


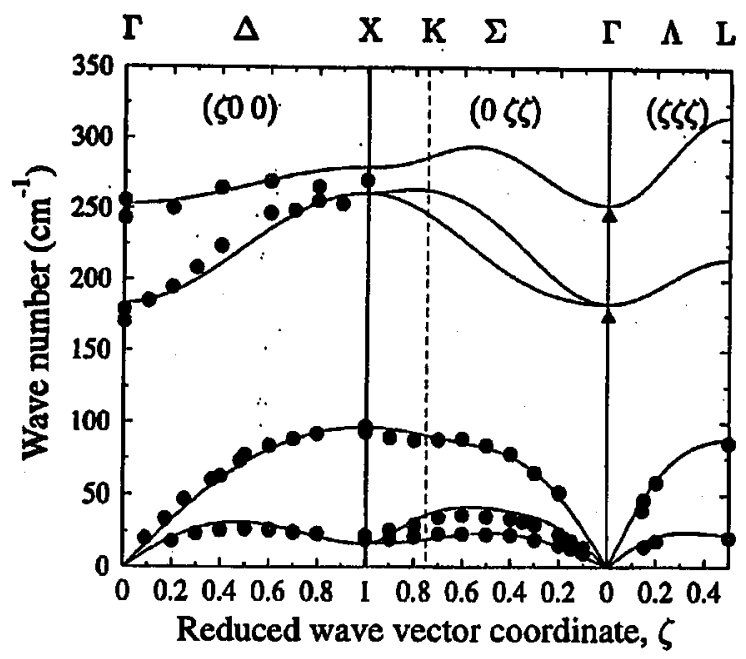

Fig. 1. Phonon dispersion along the high symmetry directions in the Brillouin zone measured for $\beta$-HgS by inelastic neutron scattering performed at $11 \mathrm{~K}$. Points represent the experimental results (for the acoustic phonons taken from [3,4], for the optical branches: present data), solid lines are the dispersion relations calculated from the non-central version of the rigid ion model [5].
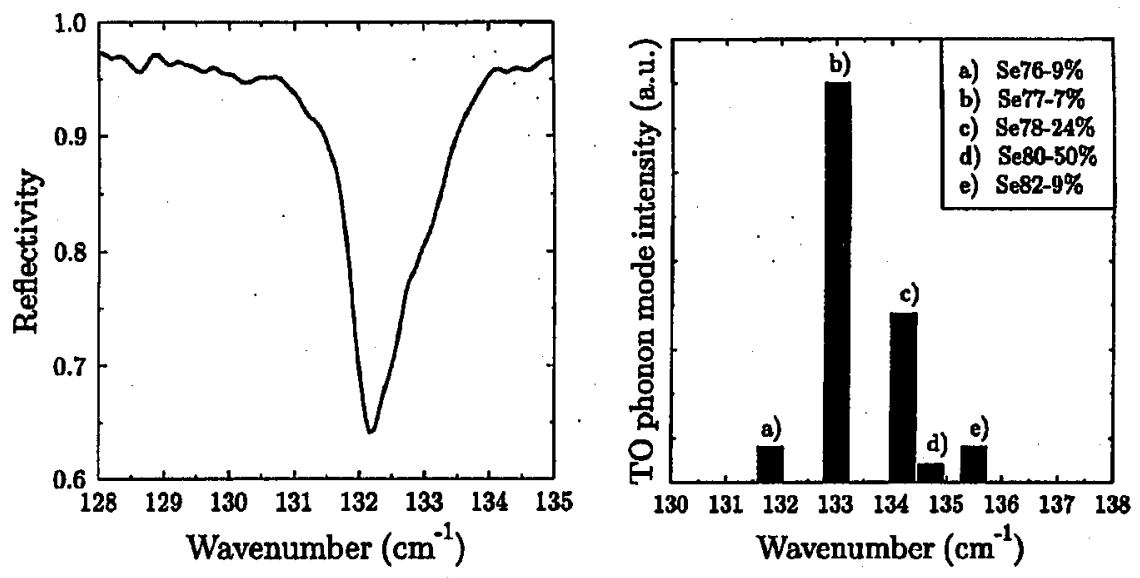

Fig. 2. Isotopic effect for TO phonon mode in HgSe. Left part - numerical simulation of TO phonon mode intensity versus frequency for $\mathrm{HgSe}$. The five principal stable Se isotope contributions are taken into account. Right part - low-temperature infrared reflectivity spectrum of $\mathrm{HgSe}$ in the vicinity of $\mathrm{TO}$ phonon mode.

the envelope given by the sum of all contributions should be asymmetric. Under the circumstances one could expect the asymmetric TO-phonon related structure observed in the infrared reflectivity spectra taken for HgSe. Figure 2b shows such a structure observed at low temperature. Experimental data confirm qualitatively 
the shape of the phonon structure, predicted by the numerical simulation. It should be stressed that this effect could not exist for $\beta$-HgS. Sulphur has only two stable isotopes: ${ }^{32} \mathrm{~S}$ and ${ }^{34} \mathrm{~S}$. Due to the small atomic mass of both isotopes the frequencies of the lattice modes corresponding to each isotope are well separated. Moreover, the natural abundance of ${ }^{34} \mathrm{~S}$ isotope is only $4 \%$, thus, the TO phonon structure observed in the reflectivity spectra of $\beta$-HgS should be symmetric in practice. As it was mentioned previously the experimental data confirm such findings. We believe that the suggested effect could be directly demonstrated by both high resolution IR reflectivity and Raman scattering spectra measured on different HgSe crystals, containing in each case only one selected selenium isotope.

As a concluding remark, we would like to state that both mercury selenide and cubic mercury sulphide appear to be very interesting materials from the point of view of the crystal lattice dynamics. High ionicity of these semiconducting compounds and significant difference of the atomic mass of constituent elements result in a large gap in the one-phonon density of states between acoustic and optical branches. They are responsible for high separation of LO and TO r.odes at the Brillouin zone center. The high dispersion observed for the optical phonon modes for $\beta-\mathrm{HgS}$ (not typical of any known II-VI or III-V semiconducting compound) results probably from the same reasons. In case of $\mathrm{HgSe}$ it is also possible to demonstrate the presence of the isotopic effects, resulting in asymmetric broadening of TO phonon related structure observed in the metallic infrared reflectivity spectra.

\section{References}

[1] K. Dybko, W. Szuszkiewicz, B. Witkowska, Deff. Diff. Forum 121-122, 41 (1995).

[2] H. Kepa, T. Giebultowicz, B. Buras, B. Lebech, K. Clausen, Phys. Scr, 25, 807 (1982).

[3] W. Szuszkiewicz, K. Dybko, E. Dynowska, J. Górecka, B. Witkowska, B. Hennion, Acta Phys. Pol. A 90, 947 (1996).

[4] W. Szuszkiewicz, K. Dybko, E. Dynowska, J. Górecka, B. Witkowska, B. Hennion, in: Proc. 23th ICPS, Berlin 1996, Eds. M. Schiffer, F. Zimmermann, Vol. 1, World Scientific, Singapore 1996, p. 253.

[5] K. Kunc, M. Balkanski, M. Nusimovici, Phys. Rev. B 12, 4346 (1975); K. Kunc: O. Holm Nielsen, Comp. Phys. Commun. 16, 181 (1979).

[6] W. Szuszkiewicz, B. Witkowska, M. Jouanne, Acta Phys. Pol. A 87, 415 (1995); W. Szuszkiewicz, B. Witkowska, M. Jouanne, M. Balkanski, Mater. Sci. Forum 182-184, 711 (1995).

[7] W. Szuszkiewicz, K. Dybko, B. Hennion, M. Jouanne, C. Julien, E. Dynowska, J. Górecka, B. Witkowska, accepted for publication in J. Cryst. Growth. 